\title{
Towards the first search for a stochastic background in LIGO data: applications of signal simulations
}

\author{
Sukanta Bose ${ }^{1}$, Bruce Allen ${ }^{2}$, Michael Landry ${ }^{3}$, Albert Lazzarini ${ }^{4}$, \\ Isabel Leonor ${ }^{5}$, Szabolcs Marka ${ }^{4}$, Tania Regimbau ${ }^{6}$, Joseph Romano ${ }^{7}$, \\ Peter Shawhan ${ }^{4}$, Daniel Sigg ${ }^{3}$ and John Whelan ${ }^{8}$ \\ ${ }^{1}$ Department of Physics and Program of Astronomy, Washington State University, Pullman, \\ WA 99164-2814, USA \\ 2 Department of Physics, University of Wisconsin-Milwaukee, 1900 E Kenwood Blvd, \\ Milwaukee, WI 53211, USA \\ ${ }^{3}$ LIGO Hanford Observatory, PO Box 159, Richland, WA 99352-0159, USA \\ ${ }^{4}$ LIGO Laboratory, California Institute of Technology, MS 18-34, Pasadena, CA 91125, USA \\ 5 Department of Physics, 1274 University of Oregon, Eugene, OR 97403-1274, USA \\ ${ }^{6}$ LIGO Project, MIT NW17-161, 175 Albany Street, Cambridge, MA 02139, USA \\ ${ }^{7}$ Department of Physics \& Astronomy, The University of Texas at Brownsville, 80 Fort Brown, \\ Brownsville, TX 78520, USA \\ ${ }^{8}$ Department of Physics, Loyola University, 6363 St Charles Avenue, Box 92, New Orleans, \\ LA 70118-6195, USA
}

Received 24 April 2003

Published 7 August 2003

Online at stacks.iop.org/CQG/20/S677

\begin{abstract}
We describe the utility of simulated-signal injection studies on earth-based gravitational-wave (GW) interferometric data toward obtaining bounds on the strength of a stochastic GW background. The existence of such a background today is predicted by several cosmological models, but with varying strengths and power spectra. Earth-based detectors, such as LIGO, will eventually achieve enough sensitivity to start constraining some of these models through these bounds. A significant part of the effort to use LIGO data to place such bounds is to estimate the efficiency of the data analysis pipeline in detecting the variety of predicted backgrounds. We took the data taking opportunity offered by the first science run at LIGO to inject simulated signals of varying strengths both in hardware as well as software. We describe here the results obtained in searching for these injection backgrounds. We discuss especially those results that either varied from the expected ones or are crucial to the search for a stochastic GW background. The reasons behind the variations are also explained.
\end{abstract}

PACS numbers: $04.80 \mathrm{Nn}, 07.05 \mathrm{Kf}, 97.80-\mathrm{d}$

(Some figures in this article are in colour only in the electronic version) 


\section{Introduction}

The three Laser Interferometer Gravitational-wave Observatory (LIGO) detectors took data simultaneously from 23 August 2001 to 9 September 2001, for the first time for scientific data analysis. These data sets of the first science run, termed S1 for short, are being studied for bounding a stochastic gravitational wave $(\mathrm{GW})$ signal of cosmological origin. One of the primary components in this quest is an estimate of the efficiency of a pair of interferometers in detecting such a background. A complete end-to-end gauge of this efficiency is possible via the injection of simulated signals in hardware that mimics the effect of a real signal by driving the end mirrors of an interferometer, and then running search codes on the corresponding detector outputs to check if the signals are recovered at the expected levels. As we discuss below, however, it is difficult to inject signals in hardware for long durations. Moreover, such injections corrupt the data and, therefore, render them unsuitable for scientific searches. Consequently, one must supplement these with software injections that involve injecting simulated signals in the output of a detector. The purpose of this paper is to describe how the hardware and software injections were performed, the studies undertaken and the results obtained in the context of stochastic signal searches in S1 data.

\section{The stochastic background}

A useful physical parameter, $\Omega$, that is often used in describing the strength of a stochastic $\mathrm{GW}$ background is the ratio of its energy density, $\rho_{\mathrm{GW}}(f)$, per logarithmic frequency interval to the critical energy density, $\rho_{0}$, required to close the universe,

$$
\Omega(f):=\frac{1}{\rho_{0}} \frac{\mathrm{d} \rho_{\mathrm{GW}}(f)}{\mathrm{d} \ln f} .
$$

Whereas this is the quantity that LIGO searches aim to bound, it is not sufficient to characterize the background that they seek. In this paper, we will assume that, on average, the stochastic GW background is isotropic, unpolarized and stationary. These properties are easily described in terms of the Fourier components of the strains produced by this background in a pair of interferometers. Let $\tilde{h}_{1}(f)$ and $\tilde{h}_{2}(f)$ be the Fourier components of the gravitational-wave strains $h_{1}(t)$ and $h_{2}(t)$ at detectors 1 and 2 , respectively. Then $\tilde{h}_{1,2}(f)$ are random variables that have zero mean and that obey [1]:

$$
\begin{aligned}
\left\langle\tilde{h}_{1}^{*}(f) \tilde{h}_{1}\left(f^{\prime}\right)\right\rangle & =\frac{3 H_{0}^{2} T}{20 \pi^{2}} \delta\left(f-f^{\prime}\right)|f|^{-3} \Omega(|f|), \\
\left\langle\tilde{h}_{2}^{*}(f) \tilde{h}_{2}\left(f^{\prime}\right)\right\rangle & =\frac{3 H_{0}^{2} T}{20 \pi^{2}} \delta\left(f-f^{\prime}\right)|f|^{-3} \Omega(|f|), \\
\left\langle\tilde{h}_{1}^{*}(f) \tilde{h}_{2}\left(f^{\prime}\right)\right\rangle & =\frac{3 H_{0}^{2} T}{20 \pi^{2}} \delta\left(f-f^{\prime}\right)|f|^{-3} \gamma(|f|) \Omega(|f|),
\end{aligned}
$$

where $H_{0}$ is the Hubble constant, $T$ is the time of observation and $\gamma$ is the overlap reduction function [2]. We will take $H_{0}=100 h_{100} \mathrm{~km}^{-1} \mathrm{~s}^{-1} \mathrm{Mpc}^{-1}$ where $h_{100}$ is a dimensionless factor included to account for the different values of $H_{0}$ quoted in the literature [3].

The above correlations are satisfied if the GW strains at each interferometer are represented by [4]

$$
\tilde{h}_{1}(f)=\sqrt{\frac{3 H_{0}^{2} T}{40 \pi^{2}}}|f|^{-3 / 2} \Omega^{1 / 2}(|f|)\left(x_{1}(f)+\mathrm{i} y_{1}(f)\right)
$$


$\tilde{h}_{2}(f)=\tilde{h}_{1}(f) \gamma(|f|)+\sqrt{\frac{3 H_{0}^{2} T}{40 \pi^{2}}}|f|^{-3 / 2} \Omega^{1 / 2}(|f|) \sqrt{1-\gamma^{2}(|f|)}\left(x_{2}(f)+\mathrm{i} y_{2}(f)\right)$,

where $x_{1}(f), y_{1}(f), x_{2}(f)$ and $y_{2}(f)$ are statistically independent real Gaussian random variables, each of zero mean and unit variance.

Without any means of distinguishing a stochastic GW in a detector from the detector's intrinsic noise, the search for such a background involves cross-correlating the outputs of a pair of detectors. As shown in [1], the detection signal in the case of an interferometric pair is

$$
\begin{aligned}
Y & =\int_{-T / 2}^{T / 2} \mathrm{~d} t \int_{-T / 2}^{T / 2} \mathrm{~d} t^{\prime} h_{1}(t) h_{2}\left(t^{\prime}\right) Q\left(t-t^{\prime}\right) \\
& =\int_{-\infty}^{\infty} \mathrm{d} f \int_{-\infty}^{\infty} \mathrm{d} f^{\prime} \delta_{T}\left(f-f^{\prime}\right) \tilde{h}_{1}^{*}(f) \tilde{h}_{2}\left(f^{\prime}\right) \tilde{Q}\left(f^{\prime}\right),
\end{aligned}
$$

where $\delta_{T}\left(f-f^{\prime}\right)$ is the finite time approximation of the Dirac delta function. Also, $Q\left(t-t^{\prime}\right)$ is the optimal filter function whose Fourier transform, $\tilde{Q}(f)$, can be shown to be [1]:

$$
\tilde{Q}(f)=\lambda \frac{\gamma(|f|) \Omega(|f|)}{|f|^{3} P_{1}(|f|) P_{2}(|f|)},
$$

where $\lambda$ is a real normalization constant and $P_{1,2}(|f|)$ are noise power-spectral densities (PSDs) of detectors 1 and 2, respectively. In the event of an exact match between the GW and filter parameters, the mean signal is

$$
\bar{Y}=\frac{3 H_{0}^{2} \lambda}{20 \pi^{2}} T \int_{-\infty}^{\infty} \mathrm{d} f \frac{\gamma^{2}(|f|) \Omega^{2}(|f|)}{f^{6} P_{1}(|f|) P_{2}(|f|)} .
$$

When $\Omega$ is taken to be a constant in the filter, we choose $\lambda$ so that $\bar{Y}=\Omega T$.

The noise is given by the variance of the statistic, $S$ :

$$
\sigma^{2}=\overline{Y^{2}}-\bar{Y}^{2} \text {. }
$$

If we assume the cross-correlation of terrestrial noises in the two detectors to be much smaller than that due to cosmological $\mathrm{GW}$, then $\sigma^{2} \approx \overline{Y^{2}}$, and one finds [1]

$$
\sigma^{2} \approx \frac{T}{4} \int_{-\infty}^{\infty} \mathrm{d} f P_{1}(|f|) P_{2}(|f|)|\tilde{Q}(f)|^{2} .
$$

Then the signal-to-noise ratio can be expressed as

$$
\begin{aligned}
\mathrm{SNR} & =\bar{Y} / \sigma \\
& \approx \frac{3 H_{0}^{2}}{10 \pi^{2}}\left[T \int_{-\infty}^{\infty} \frac{\mathrm{d} f f^{-6} \gamma^{2}(|f|) \Omega^{2}(|f|)}{P_{1}(|f|) P_{2}(|f|)}\right]^{1 / 2} .
\end{aligned}
$$

\section{Data analysis}

The search for a stochastic GW background in LIGO data comprises first selecting $900 \mathrm{~s}$ long simultaneous strain data sets in a pair of detectors. Each $900 \mathrm{~s}$ job is then subdivided into 90 s segments, which are then windowed, zero-padded and converted to the Fourier domain using fast Fourier transform (FFT) algorithms [5]. Let $I$ denote the $900 \mathrm{~s}$ long sets and $J$ the $90 \mathrm{~s}$ long segments within each set. Then, the mean of the cross-correlation (CC) statistic computed on set $I$ can be expressed as

$$
\bar{Y}_{I}=\frac{1}{10} \sum_{J=1}^{10} Y_{I J}
$$


and its standard deviation as

$$
\sigma_{I}=\left[\frac{1}{9} \sum_{J=1}^{10}\left(Y_{I J}-\bar{Y}_{I}\right)^{2}\right]^{1 / 2} .
$$

The weighted average of the cross-correlation statistic over $M 900 \mathrm{~s}$ data sets is

$$
\bar{Y}=\frac{\sum_{I=1}^{M} \lambda_{I} \bar{Y}_{I}}{\sum_{I=1}^{M} \lambda_{I}},
$$

where the optimal weights can be shown to be $\lambda_{I}=\sigma_{I}^{-2}[1]$. For $\Omega(f)=\Omega_{0}=$ const, the point estimate of $\Omega_{0}$ can then be expressed as a function of $\bar{Y}$ :

$$
\hat{\Omega}_{0}=\bar{Y} / T \text {, }
$$

with a standard error equal to

$$
\hat{\sigma}:=\frac{1}{T} \frac{1}{\sqrt{10}} \frac{\left(\sum_{I=1}^{M} \lambda_{I}^{2} \sigma_{I}^{2}\right)^{1 / 2}}{\sum_{I=1}^{M} \lambda_{I}} .
$$

In principle, the point estimate, $\bar{Y}$, computed on the observed data (and, therefore, $\hat{\Omega}_{0}$ given in equation (14)) should be expected to agree with that given by the theoretical mean in equation (7). In practice, however, the two could be different owing to the likely presence of non-Gaussianity in the detector noises, incompleteness in the knowledge of the transfer functions that are used in translating the GW strain in a detector's arms to the data output of that detector and the effects of conditioning the data while executing a search. To check how much these factors affect our results, we do simulated signal injections and look for deviations between the results of searches conducted on the injection data and those expected theoretically. This is the topic of discussion in the following section.

\section{Injection of simulated signals}

Studies such as upper limits are partly based on the Monte Carlo simulations with signals injected into the datastream in software. A more accurate estimate of detection efficiency can be obtained by comparing these signals to the ones injected into the detectors. The latter are called 'hardware' injections and serve to test the entire detection pipeline, right from the movement of the mirrors to analysing the interferometric output.

There are several advantages of injecting simulated signals and studying how they affect a search statistic. First, they serve as a check on the accuracy of the calibration methods being applied to infer the strain in an interferometer. Second, they can gauge the timing accuracy of the strain data. Third, they aid in the study of inter-detector cross-correlated noise. Finally, given that stochastic signal injections are of long duration, these injections allow us to learn about systematic errors that build up on long timescales.

Typically, a source model is used to simulate the signal at a detector. For instance, various cosmological models could be used to predict the power spectrum of the signal due to a stochastic background of cosmological gravitational waves. At the current level of detector sensitivities, it is unlikely that we can find a stochastic signal even if it were with a strength predicted by some of the most optimistic cosmological models [6, 7], and in spite of a year-long integration time. Nevertheless, with ever improving sensitivities, we can start obtaining useful observational upper bounds on the background and, therefore, begin constraining theoretical models.

In order to test the ability of our stochastic data analysis pipeline, one can imagine injecting a signal (hardware or software) over the full length of a data set and then applying 
search codes to look for the signal. The longer the run, the tougher the challenge of simulating and injecting the signal, especially in hardware. Moreover, whereas it is possible, in principle, to do such injections in software without corrupting the original copy of the data, hardware injections necessarily alter the data and, therefore, render the data unsuitable to do science with. Nevertheless, given the utility of hardware injections, we decided to perform them immediately preceding and following S1 at LIGO, and for durations relatively shorter than the run itself.

Hardware injections need to be of short duration owing to the fact that long injections can build up non-linearities within the arm cavities that eventually drive the detector out of lock. Shorter injections, on the other hand, require that the strength of the simulated signal be large enough to allow detection with the shortened integration times. The problem with this is that large signals can also drive a detector out of lock. For S1, it was found that signals with an SNR of $\mathcal{O}(10)$ and duration of 1024 s could be injected without breaking lock. What kind of $\Omega$ did these signals correspond to? We address this question below.

\subsection{Determining injection scales}

To produce simulated signals, we first create two reference time-series, $h_{1}(t)$ and $h_{2}(t)$, by inverse-Fourier transforming the frequency components in equation (3), after setting $\Omega$ equal to 1 there. As shown by equation (3), this does not require knowledge of the noise PSD of either detector. Indeed, in different pairs of interferometers the same pair of signal strains will produce varying SNRs. To inject simulated signals with a given SNR, one uses equation (10) to estimate the correct $\Omega$ corresponding to the noise PSDs of the two detectors. This is especially easy when $\Omega$ is taken to be a constant, such as here. Let the value of the required $\Omega$ be $\Omega_{0}$. Then, the corresponding strains are the reference strains (i.e., strains with $\Omega=1$ ) scaled up by $\sqrt{\Omega_{0}}$. The injection scripts allow for operating with the same reference waveforms (created with $\Omega=1$ ), but injected with different scales $\sqrt{\Omega_{0}}$. While allowing us to perform the injections, this facility saves on both the computing time and the disk space required for creating individual time-series for a variety of $\Omega_{0}$.

As shown by equation (10), for weak signals (i.e., small $\Omega$ ), the SNR scales linearly with $\Omega$. For strong signal injections, the approximate expression for the SNR given in equation (10) does not hold: the simulated signal itself becomes a significant fraction of the total detector strain and, therefore, the actual SNR asymptotes to a constant value for large $\Omega$. This behaviour of the SNR as a function of $\Omega$ is shown in figure 1 for the Harrison-Zel'dovich spectrum, with $\Omega=\Omega_{0}$. This figure also suggests a wide range of values of $\Omega_{0}$ that correspond to SNRs from less than 1 to a little above 10 . For S1 injection, the choices of $\Omega_{0}$ were based on such a range of expected SNRs, and are listed in table 1.

Codes are available in the LIGO Algorithm Library (LAL) [8] for simulating stochastic GW signals. A simulated signal is a timeseries with units of strain. Injecting it in hardware or software requires convolving it with detector transfer functions to mimic the effect of the interaction between an astrophysical GW and the detector. We now describe these procedures below.

\subsection{Hardware injections}

Hardware injection of a simulated signal uses the excitation engine in a detector's global diagnostic system (GDS) to swing the end test mass(es) (or end mirror(s)) by applying a time-varying voltage to the driving coil of the mirror(s). A simulated signal is used by the injection script to generate the driving coil voltage [9]. Mimicking the effect of an impinging 


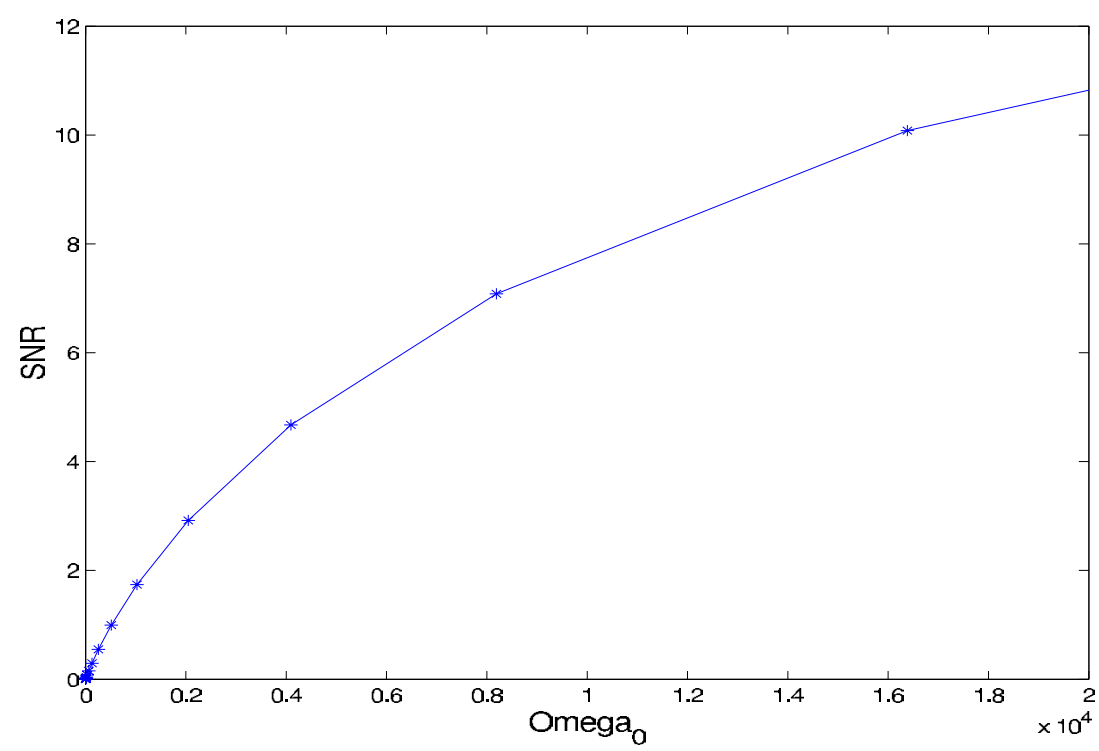

Figure 1. Plot of the expected SNR versus $\Omega_{0}$ for simulated sensitivities of a Hanford-Livingston detector pair.

Table 1. The values of $\Omega_{0}$ and the corresponding scales of the signals that were injected in hardware (and software) in the H2-L1 pair during S1.

\begin{tabular}{cl}
\hline$\Omega_{0}$ & Injection scale \\
\hline 625 & 25 \\
3906.25 & 62.5 \\
24414.0625 & 156.25 \\
\hline
\end{tabular}

GW as closely as possible requires moving the end test masses in both the $X$ and $Y$ arms in tandem to simulate a differential mode motion. A limited scheme involves injection of the simulated signal in only one of the two arms (say, the $X$ arm). In either case, it is required that the simulated signals, $\tilde{h}_{1,2}(f)$, be convolved with the pendulum transfer function of the end test mass(es). A pendulum transfer function [10] depends on its resonance frequency, $f_{0}$, and its amplitude at $f=0, f_{0}$, the often termed DC amplitude, $A_{0} .{ }^{9}$ In the frequency domain, this transfer function is defined as

$$
T(f)=\frac{f^{2}}{f_{0}^{2}} A_{0}^{-2} .
$$

Thus, the injected signals, $o_{1,2}(t)$, are the inverse Fourier transforms of the following quantities:

$$
\tilde{o}_{1,2}(f):=T_{1,2}(f) \tilde{h}_{1,2}(f),
$$

for detectors 1 and 2 , respectively.

9 The pendulum frequency and DC amplitude [10] and, therefore, the pendulum transfer function may vary from one science run to another. These data are, therefore, announced beforehand on the calibration homepage of the detectors. Typically, the pendulum DC amplitude is supplied to the theorist in units of nanometre per count. But equation (16) assumes that the length of the interferometer has been accounted for in order to derive its dimensionless relative, $A_{0}$, in strain per count. 


\subsection{Software injections}

This type of injection is done into the detector output, which is whitened. These data are a time series and have units of count. Thus, the simulated signals, $\tilde{h}_{1,2}(f)$, must be convolved with a detector's whitening function before they can be injected into the respective detector outputs ${ }^{10}$. The point of injection is decided by the objective of an analysis. For example, if one wants to study the effect of regression of line-harmonics on a potential GW signal, then one injects the whitened simulated GW signal into the whitened detector output before the lines are regressed, say, in the dataConditioningAPI ${ }^{11}$.

If the (frequency domain) whitening functions for detectors 1 and 2 are $\tilde{W}_{1,2}(f)$ respectively, then the injected strains are also whitened using the same functions:

$$
\tilde{o}_{1,2}(f):=\tilde{W}_{1,2}(f) \tilde{h}_{1,2}(f) .
$$

The above whitened frequency series are inverse Fourier transformed to obtain the real timeseries $o_{1,2}(t)$, which are then injected in software into the whitened detector outputs.

\section{Results}

The application of our search codes on the detector output containing the hardware injections allowed us to validate our data analysis pipeline by demonstrating the ability to detect a coincident excitation of a pair of detectors by simulated waveforms that mimic the properties of the real background, albeit with a larger SNR. We now discuss the specifics of the results we obtained.

\subsection{Point estimates}

The plots of the point estimates of $\Omega_{0}$ for the hardware-injection data are shown in figure 2 . Table 2 summarizes the search results for each of the hardware injections. Note that in each case, the actual $\Omega_{0}$ falls within $90 \%$ confidence limit (CL) error bars of the (negative of the) point estimates. The odd aspect of these H2-L1 injections is that the point estimate is the negative of a value close to the injected one. A priori there can be several reasons for this observation. One possibility is that the simulation codes had a relative sign error between the two theoretical strain expressions. Another possibility is that the overlap reduction function being used by the search codes is erroneous up to a sign.

A thorough test of the codes and other aspects of the simulations were conducted to address this issue. Indeed, the same codes were used (but with whitening functions instead of the pendulum transfer functions) to simulate signals for software injections. These signals were then injected on the S1 'playground' data set aside for testing purposes. The point estimates on the software-injection data did turn out positive. These are plotted in figure 3 . The software-injection results helped in ruling out some of the possible explanations for the negative point estimates found in hardware injections, such as those mentioned above. This and several other tests showed that the sign error, in fact, originated from the different calibration function signs being used at the detector sites in Livingston and Hanford. The cure for this error is simple. It involves tracking the phase of the calibration function accurately at the two sites. This tracking has been implemented since.

\footnotetext{
${ }^{10}$ The whitening functions are also made available on the calibration homepage of the detectors.

11 The dataConditioningAPI is a toolbox in the LIGO data analysis system; it comprises several modules for conditioning the data. It includes, among others, modules for computing the running mean and variance, as well as for resampling the data.
} 

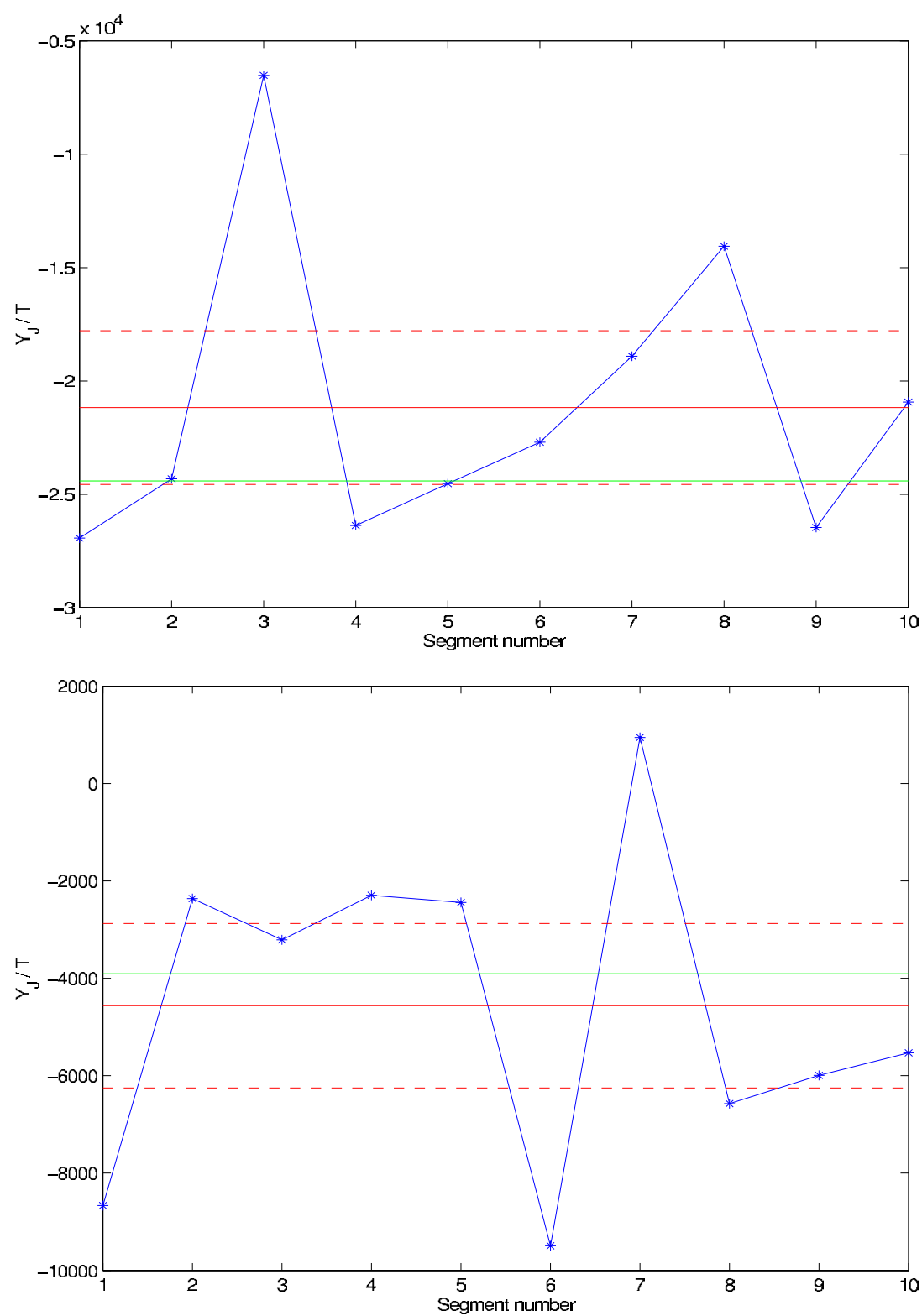

Figure 2. Plot of the $10 \mathrm{CC}$ statistic values (shown by asterisks and connected by a zig-zag curve), the point estimate of $\Omega_{0}$ (dark solid line), 90\% CL error bars (dashed lines), and the negative of the actual value of $\Omega_{0}$ (faint solid line) of simulated signals injected in hardware simultaneously in $\mathrm{L} 1$ and $\mathrm{H} 2$ during $\mathrm{S} 1$. Whereas $\Omega_{0}$ is 24414.0625 in the top plot, it is 3906.25 in the bottom plot.

Table 2. The values of hardware injected $\Omega_{0}$, and the point estimates of $\Omega_{0}$ obtained by applying the search codes to the injected data in a detector pair during $\mathrm{S} 1$.

\begin{tabular}{lcl}
\hline Detector pair & Injected $\Omega_{0}$ & Point estimate \\
\hline H2-L1 & 3906.25 & $-4.562638 \times 10^{3}$ \\
H2-L1 & 24414.0625 & $-2.117348 \times 10^{4}$
\end{tabular}



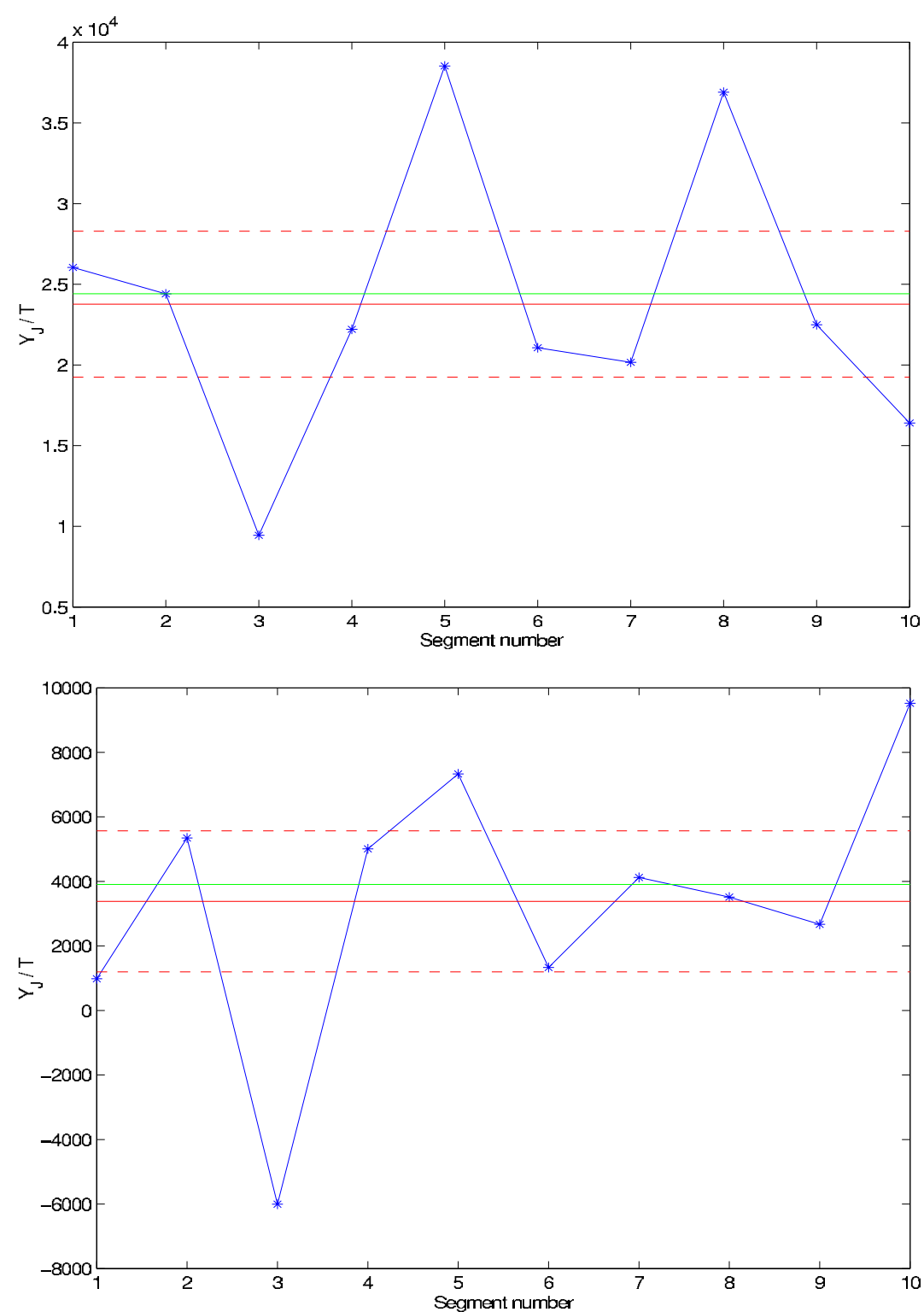

Figure 3. Plot of the $10 \mathrm{CC}$ statistic values (shown by asterisks and connected by a zig-zag curve), the point estimate of $\Omega_{0}$ (dark solid line), 90\% CL error bars (dashed lines) and the actual value of $\Omega_{0}$ (faint solid line) of simulated signals injected in software simultaneously in L1 and $\mathrm{H} 2$ during S1. Whereas $\Omega_{0}$ is 24414.0625 in the top plot, it is 3906.25 in the bottom plot.

\subsection{Time-shift analysis}

Apart from obtaining point estimates for zero time-shift between the data sets from a pair of detectors, our codes also have the functionality to compute estimates as a function of a nonvanishing time-shift. The expression for the cross-correlation statistic given in equation (5) can be generalized to the case where the data from one of the detectors are time-shifted by an 

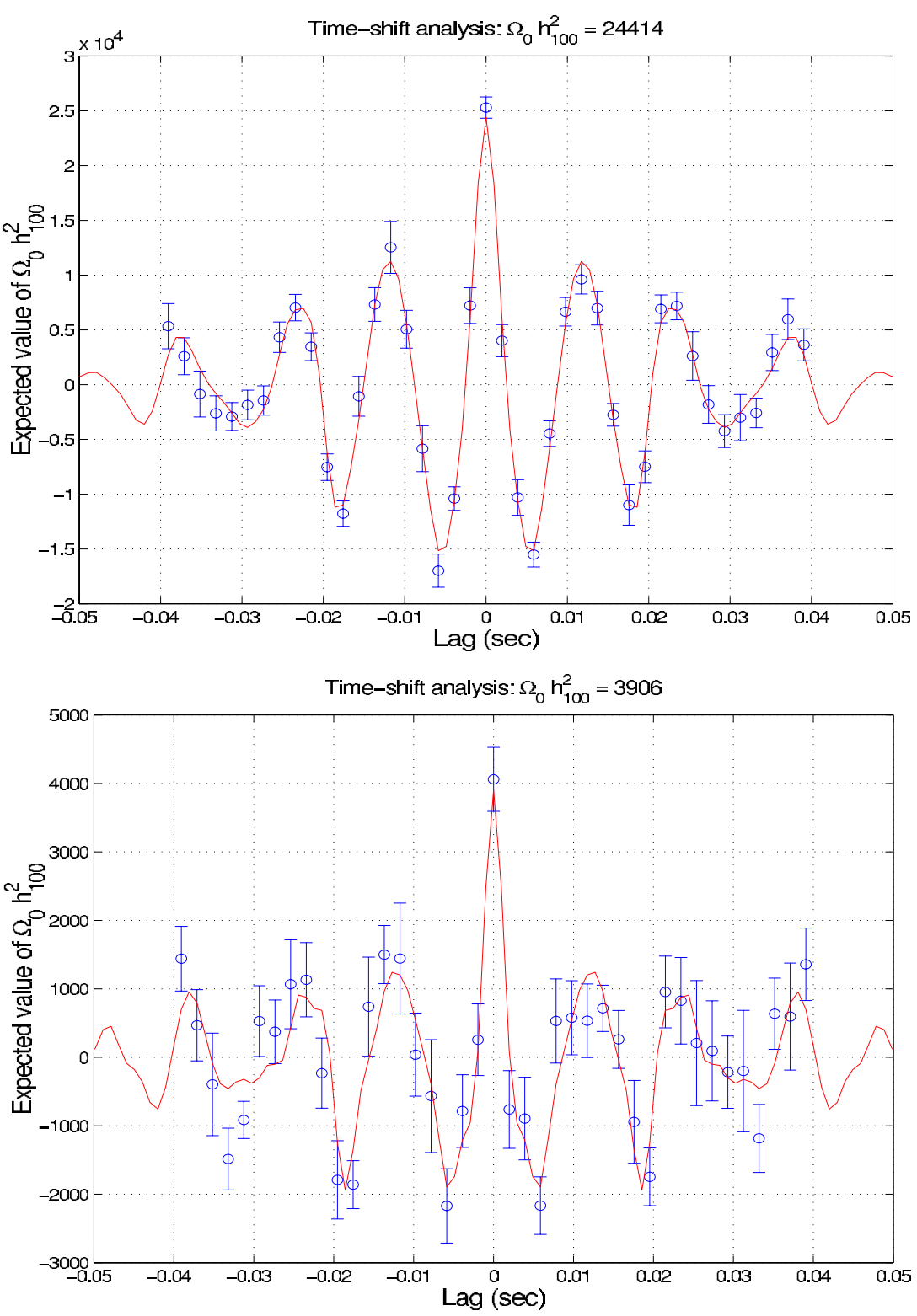

Figure 4. Plots of the time-shift analysis of a simulated signal injected in software in L1 and H2 playground data from $S 1$. The top plot is for data with injected $\Omega_{0}=24414.0625$, whereas the bottom plot is for data with injected $\Omega_{0}=3906.25$. The blue circles are the point estimates of $\overline{Y(\tau)} / T$ measured at discrete values of the time-shift, $\tau$. Its value at $\tau=0$ gives the estimated value of $\Omega_{0}$. The continuous curve shows the expected behaviour.

amount $\tau$ with respect to the data from the other. The resulting statistic is

$$
\begin{aligned}
Y(\tau) & =\int_{-T / 2}^{T / 2} \mathrm{~d} t \int_{-T / 2}^{T / 2} \mathrm{~d} t^{\prime} h_{1}(t+\tau) h_{2}\left(t^{\prime}\right) Q\left(t-t^{\prime}\right) \\
& =\int_{-\infty}^{\infty} \mathrm{d} f \int_{-\infty}^{\infty} \mathrm{d} f^{\prime} \delta_{T}\left(f-f^{\prime}\right) \tilde{h}_{1}^{*}(f) \tilde{h}_{2}\left(f^{\prime}\right) \tilde{Q}\left(f^{\prime}\right) \mathrm{e}^{\mathrm{i} 2 \pi f^{\prime} \tau} .
\end{aligned}
$$


The expected value of the above quantity is computed by using equation (2) to be

$$
\overline{Y(\tau)}=\frac{3 H_{0}^{2} T}{20 \pi^{2}} \int_{-\infty}^{\infty} \mathrm{d} f|f|^{-3} \gamma(|f|) \Omega(|f|) \tilde{Q}(f) \mathrm{e}^{\mathrm{i} 2 \pi f \tau} .
$$

The plots of $\overline{Y(\tau)}$ for two different values of $\Omega=\Omega_{0}$ are shown by the continuous curves in figure 4 . These plots show a remarkably good agreement with the estimated values computed on injected data (shown by the circles). Note that the expected values (shown by the continuous curve) fall within the error bars of almost all the measured points. Moreover, the resolution of the plots rules out the presence of timing errors of more than several microseconds.

\section{Discussion}

While the studies we have conducted so far with simulated signals are not as exhaustive as those associated with the Monte Carlo simulations, they have nevertheless served a useful purpose in unravelling errors in some parts of our data analysis pipeline or validating the functioning of others. These studies were also limited in that they explored only the 'flat' Harrison-Zel'dovich spectrum, with $\Omega=$ const. Future studies will aim to overcome these shortcomings.

\section{Acknowledgments}

The authors gratefully acknowledge the support of the United States National Science Foundation for the construction and operation of the LIGO Laboratory, and for the work discussed in this paper.

\section{References}

[1] Allen B and Romano J D 1999 Phys. Rev. D 59102001 Preprint gr-qc/9710117

[2] Flanagan E 1993 Phys. Rev. D 482389

[3] Freedman W et al 2001 Astrophys. J. 55347

[4] Allen B 2000 'GRASP: a data analysis package for gravitational wave detection' University of WisconsinMilwaukee Distribution website URL: http://www.lsc-group.phys.uwn.edu/ ballen/grasp-distribution

[5] For an explanation on what the FFT algorithms are and how they work see, e.g.Press W H, Teukolsky S A, Vellerling W T and Flannery B P 1993 Numerical Recipes in C 2nd edn (Cambridge: Cambridge University Press)

[6] Allen B 1995 The stochastic gravity-wave background: sources and detection Proc. Les Houches School on Astrophysical Sources of Gravitational Waves ed J A Marck and J P Lasota (Cambridge: Cambridge University Press) pp 373-417

[7] Magiore M 2000 Gravitational wave experiments and early universe cosmology Phys. Rep. 331 283-367

[8] The Concurrent Version Repository of the LIGO Algorithm Library. Webpage:http://www.lscgroup.phys.uwm.edu/lal/

[9] Shawhan P and Sigg D 2002 The Signal Injection Handbook LIGO Technical Document LIGO-T020072-00-D

[10] Saulson P 1994 Fundamentals of Interferometric Gravitational Wave Detectors (Singapore: World Scientific) 